\title{
Nordiques
}

33 | 2017

La transition des villes nordiques : quelles innovations territoriales en périphérie?

\section{La dimension spatiale de l'innovation dans les régions périphériques nordiques}

\section{Alexandre Dubois}

\section{OpenEdition}

\section{Journals}

Édition électronique

URL : https://journals.openedition.org/nordiques/2520

DOI : 10.4000 /nordiques. 2520

ISSN : 2777-8479

Éditeur :

Association Norden, Bibliothèque de Caen la mer

Édition imprimée

Date de publication : 1 mai 2017

Pagination : 11-24

ISBN : 978-2-9544654-9-4

ISSN : $1761-7677$

\section{Référence électronique}

Alexandre Dubois, «La dimension spatiale de l'innovation dans les régions périphériques nordiques », Nordiques [En ligne], 33 | 2017, mis en ligne le 19 novembre 2021, consulté le 02 février 2022. URL : http://journals.openedition.org/nordiques/2520 ; DOI : https://doi.org/10.4000/nordiques.2520 


\section{La dimension spatiale de l'innovation dans les régions périphériques nordiques}

Alexandre Dubois*

\section{RÉSUMÉ}

Cet article se propose d'explorer la dimension spatiale des problématiques contemporaines $d u$ développement régional dans les zones périphériques nordiques. Historiquement, le développement de ces régions s'est fait sur la base de l'exploitation des abondantes ressources naturelles disponibles, telles que les minerais (fer, cuivre...) ou le bois. Dans une première partie, l'article propose d'introduire un cadrage conceptuel permettant de mieux apprébender la complexité des systèmes de relations entretenues par les acteurs de ces régions. Dans une deuxième partie, l'exemple d'une PME d'une localité minière du Nord de la Suède est utilisé afin d'illustrer comment ce cadrage permet de comprendre les dimensions relationnelle et spatiale de ces processus. Enfin, dans la dernière partie, l'article propose de discuter les récents exemples venus de l'implémentation de la stratégie de la "spécialisation intelligente " permettant d'exposer les défis du développement territorial, notamment en ce qui concerne l'équilibre à trouver entre la gouvernance des ressources naturelles et l'inclusion de ces régions dans l'économie de la connaissance, au travers de la gouvernance territoriale.

\section{ABSTRACT}

This article aims to review and discuss the spatial dimension of contemporary processes of economic development in the northern sparsely populated areas of the Nordic countries. Historically, these regions have developed thanks to the exploitation of its abundant natural resources, such as ores (iron, copper) or timber. In its first part, the article introduces a conceptual framework that gives a more complete and systematic understanding of the complex system of relations that actors in these regions are engaged in. The article then continues by illustrating this framework using the example of the development trajectory of a small firm located in a mining area of north Sweden. Finally, the article discusses recent examples from the implementation of Smart Specialisation strategies in Nordic peripheral regions. The article emphasizes the role of territorial governance as a way to balance the challenges and opportunities inherent to the sustainable management of natural resources and the promotion of the Knowledge Economy.

* Alexandre Dubois est chercheur en développement rural à l'université suédoise des sciences agricoles à Uppsala. Ingénieur, aménageur et géographe de formation, il a précédemment travaillé au sein de l'Institut Nordregio sur les questions du développement territorial dans les zones périphériques nordiques en lien avec la politique européenne de cohésion. 


\section{INTRODUCTION}

L'ambition de cet article est de donner une vision d'ensemble des processus contemporains de développement et d'innovation dans les régions septentrionales de la Finlande, de la Norvège et de la Suède, que l'on peut caractériser comme étant à la fois périphériques géographiquement, c'est-à-dire éloignées des grandes métropoles nationales (Helsinki, Oslo et Stockholm) et continentales, et faiblement peuplées.

Du point de vue économique, l'exploitation des abondantes ressources naturelles, comme le bois ou les minerais, a permis l'essor de l'économie régionale et a été un grand pourvoyeur d'emplois, plutôt bien rémunérés, pour les populations locales. Historiquement, cette économie de ressources a aussi permis le développement de l'État providence nordique moderne tel que nous le connaissons. Ainsi, ces territoires qui font partie des régions nordiques, à l'exception des capitales, ayant un niveau de PIB par habitant des plus élevés ${ }^{1}$, ont été au cour du projet national de développement économique.

Malgré cela, les territoires septentrionaux ont été marginalisés dans la construction sociale et politique du territoire national. Dans ce cadre-là, l'exemple de la Suède est particulièrement pertinent. En effet, Nilsson et Lundgren affirment que dans la rhétorique politique nationale, la périphérie septentrionale est souvent perçue comme un "problème $"^{2}$. Ils expliquent notamment que le "problème » est moins le fait des qualités caractéristiques de ces territoires que du changement du système de valeurs du reste de la société suédoise qui a modifié son lien affectif avec la périphérie. Le style de vie urbain, perçu comme plus progressiste et moderne, est ainsi devenu la norme, notamment relayé par les médias nationaux ${ }^{3}$. Dans ce contexte, la périphérie a été associée à des représentations négatives, telles que le chômage ou la dépopulation, dans l'imaginaire collectif. Cette périphéralisation des territoires septentrionaux est donc le produit de processus sociopolitiques résultant de l'urbanisation et de la polarisation du territoire national au profit des zones urbaines du Sud.

Pour autant, les défis actuels du développement de ces régions sont bien réels. L'exploitation des ressources naturelles est toujours très profitable

1 Erik Gløersen, Alexandre Dubois, Andrew Copus, Carsten Schürmann, " Northern Peripheral, Sparsely Populated Regions in the European Union and in Norway ", Nordregio Report, 2, 2006.

2 Bo Nilsson, Anna Sofia Lundgren, "Logics of Rurality : Political Rhetoric about the Swedish North ", Journal of Rural Studies, 37, 2015, p. 85-95.

3 Madeleine Eriksson, "(Re)producing a "Peripheral" Region : Northern Sweden in the News ", Geografiska Annaler. Series B, Human Geography, 90, 4, 2008, p. 369-388. 
économiquement, même si elle tend à moins bénéficier aux populations locales, dû à leur automatisation. Le principal défi est lié à la gouvernance de ces ressources naturelles qui doit faire interagir de multiples acteurs aux intérêts parfois divergents, allant du niveau local au niveau global, et leur inclusion dans les stratégies à long terme de développement régional. Cet article propose au lecteur de se pencher sur la problématique de la gouvernance territoriale comme enjeu central pour le développement durable régional autour de deux axes majeurs : le lien que ces régions et leurs acteurs entretiennent avec la globalisation et les processus d'implémentation par ces régions des stratégies régionales de l'innovation dites de la "spécialisation intelligente".

\section{ConteXte de L'ÉTUde}

Le but de cette étude est donc avant tout de montrer que cette " marge géographique " n'est pas marginale, au sens premier du terme, mais plutôt se constitue comme espace-interface ${ }^{4}$ au confluent de multiples systèmes, mondiaux, nationaux ainsi que régionaux. Historiquement, le développement des territoires septentrionaux nordiques s'est forgé au gré de relations entre les acteurs locaux et ceux de centres de décisions qu’ils soient publics (État) ou privés (entreprises multinationales). En faisant un raccourci, on pourrait donc estimer que le développement territorial est le résultat de relations de dépendance du local vis-à-vis d'acteurs plus centraux d'un point de vue décisionnel. Ainsi, même si les études concernant les processus de globalisation dans les zones rurales et périphériques ont mis en avant la plus grande capacité d'action et d'adaptation des acteurs locaux et ainsi la possibilité pour ces acteurs de bénéficier des potentialités liées à la mondialisation ${ }^{5}$, le discours proposé est soit diffus, parlant de mondialisation comme d'un processus relationnel "générique ", soit anecdotique, se fondant sur quelques «bonnes pratiques » ou «success stories » qui, bien que très informatives, ne permettent pas de comprendre d'une manière systématique comment ces trajectoires de développement se matérialisent.

De ce constat est venue l'idée de développer la grille analytique $\mathrm{ARTE}^{6}$ présentée dans la première partie de cet article. Cette grille peut être utilisée pour comprendre la trajectoire de développement d'acteurs locaux, que ce soient des

4 Erik Gløersen, La Finlande, la Norvège, la Suède face au projet d'une Europe polycentrique. La centralité à la marge de l'Europe, Rennes, Presses universitaires de Rennes, 2012.

5 Michael Woods, "Engaging the Global Countryside : Globalization, Hybridity and the Reconstitution of Rural Place ", Progress in Human Geography, 31, 4, 2007, p. 485-507.

6 ARTE est l'acronyme des termes en anglais Agglomeration, Regionalisation, Translocalisation et Europeanisation. 
collectivités territoriales (régions, municipalités), mais aussi des acteurs privés (SME, PME) ou institutionnels (universités). Dans la seconde partie de cet article, nous proposons d'appliquer cette grille d'analyse à l'exemple d'une petite entreprise du Nord de la Suède spécialisée dans la fabrication de radars pour l'exploration souterraine et ainsi de mieux comprendre les aspects relationnels de sa trajectoire historique de développement.

Le but de la grille d'analyse est de mieux faire ressortir la dimension spatiale des processus d'innovation et de développement dans les régions périphériques. Cette dimension spatiale non seulement permet de comprendre comment les acteurs régionaux se positionnent au travers des réseaux transnationaux de coopération, mais aussi comment ces trajectoires nécessitent une reconfiguration des relations infrarégionales. C'est ce propos que nous essaierons d'illustrer dans la troisième partie de cet article, au travers de notre analyse des processus actuels d'implémentation de la stratégie dite de la "spécialisation intelligente » dans les régions périphériques nordiques.

\section{LES DIMENSIONS RELATIONNELLE ET SPATIALE DU « DÉVELOPPEMENT SEPTENTRIONAL »}

$\mathrm{Au}$ premier abord, la position excentrée des périphéries nordiques vis-à-vis des zones métropolitaines nordiques et continentales laisse penser à une certaine forme d'isolement de ces territoires et de leurs acteurs. Au contraire, la forte internationalisation des industries extractives fait que les régions périphériques nordiques ont été très tôt confrontées à la mondialisation. Le développement économique localisé se fondant essentiellement sur l'exploitation de ressources naturelles est tellement caractéristique de ce type de zones géographiques qu'il en porte le nom, c'est-à-dire le "développement septentrional " (northern development en anglais). Le développement septentrional promeut la mise en valeur des ressources locales par la mobilisation de moyens externes (connaissances, maind'œuvre, capitaux, etc.). Ces "périphéries des ressources" (resource peripheries en anglais) sont donc dépendantes d'agents externes, autant économiques que politiques, qui influent sur les marchés des commodités naturelles. Bien que les ressources naturelles exploitées soient différentes d'un pays à l'autre (pêche et énergie pour la Norvège, pâte à papier et bois pour la Finlande et extraction

7 Mel Watkins, "Staples Redux ", Studies in Political Economy, 79, 2009, p. 213-226 ; Dean B. Carson, Prescott Ensign, Rasmus Ole Rasmussen, Andrew Taylor, « Perspectives on "Demography at the Edge" ", in Demography at the Edge. Remote Human Populations in Developed Nations, D. B. Carson, R. O. Rasmussen, P. Ensign, L. Huskey et A. Taylor (dir.), Farnham, Ashgate, 2011, p. 3-20. 
minière et bois pour la Suède ${ }^{8}$ ), la problématique engendrée reste en beaucoup de points similaire. En effet, les opérateurs " globalisés » exploitant ces ressources naturelles sont autant des entreprises multinationales, souvent originaires de pays à forte tradition minière, comme le Canada ou l'Australie, que des grandes entreprises à actionnariat public (État et/ou collectivités territoriales), comme LKAB ou Boliden en Suède. Ainsi, il apparaît, à l'instar de l'exemple suédois, que le développement du Nord du pays a été longtemps coordonné par le gouvernement national par le biais de compagnies nationalisées dans ces industries 9 . Au-delà de cette vulnérabilité systémique, la capacité des acteurs de terrain à compenser et atténuer les effets négatifs des cycles successifs d'expansion et effondrement (booms-and-busts en anglais) qui sont caractéristiques de ces industries grâce aux initiatives locales a été le sujet d'études passées ${ }^{10}$.

Peut-on parler pour autant d'une " malédiction " des ressources naturelles ? Après tout, leur exploitation a bénéficié, d'une manière ou d'une autre, aux multiples acteurs qui y prennent part : État, multinationales, collectivités territoriales. La problématique relève plutôt du fait que l'exploitation des ressources naturelles polarise fortement le paysage économique régional et limite la capacité de ses acteurs de se projeter à plus long terme vers d'autres formes de développement. Ainsi, pour les "périphéries de ressources ", le risque est que ces territoires deviennent économiquement et politiquement " piégés " par une dépendance trop importante aux exportations de ressources naturelles peu ou pas transformées ${ }^{11}$. Ainsi, comprendre les tenants d'un développement territorial " post-ressources naturelles » dans ces régions périphériques, notamment en utilisant une approche de prospective territoriale comme nous le verrons dans la dernière partie de cet article, nécessite de mieux pouvoir appréhender comment les acteurs se positionnent dans les processus contemporains de globalisation. La grille d'analyse $\mathrm{ARTE}^{12}$ introduite dans le reste de cette section permet de mettre en avant quatre processus majeurs aux spatialités et mécanismes relationnels spécifiques.

8 Erik Gløersen, Alexandre Dubois, Johanna Roto, Rasmus Ole Rasmussen, José Sterling, «Development Perspectives for the NSPA : Opportunities and Challenges », Nordregio, 3, 2009.

9 Dean B. Carson, Doris A. Carson, Gabriella Nordin, Peter Sköld, «Lessons from the Arctic Past : The Resource Cycle, Hydro Energy Development, and the Human Geography of Jokkmokk, Sweden », Energy Research \& Social Science, 16, 2016, p. 13-24.

10 Adam Wellstead, "The (Post) Staples Economy and the (Post) Staples State in Historical Perspective ", Canadian Political Science Review, 1, 1, 2007, p. 8-25.

11 Dean B. Carson, Doris A. Carson, Gabriela Nordin, Peter Sköld, op. cit., p. 3.

12 Alexandre Dubois, Dean B. Carson, "Placing Northern Development : The Case of North Sweden ", Local Economy, 31, 7, 2016, p. 795-811. 


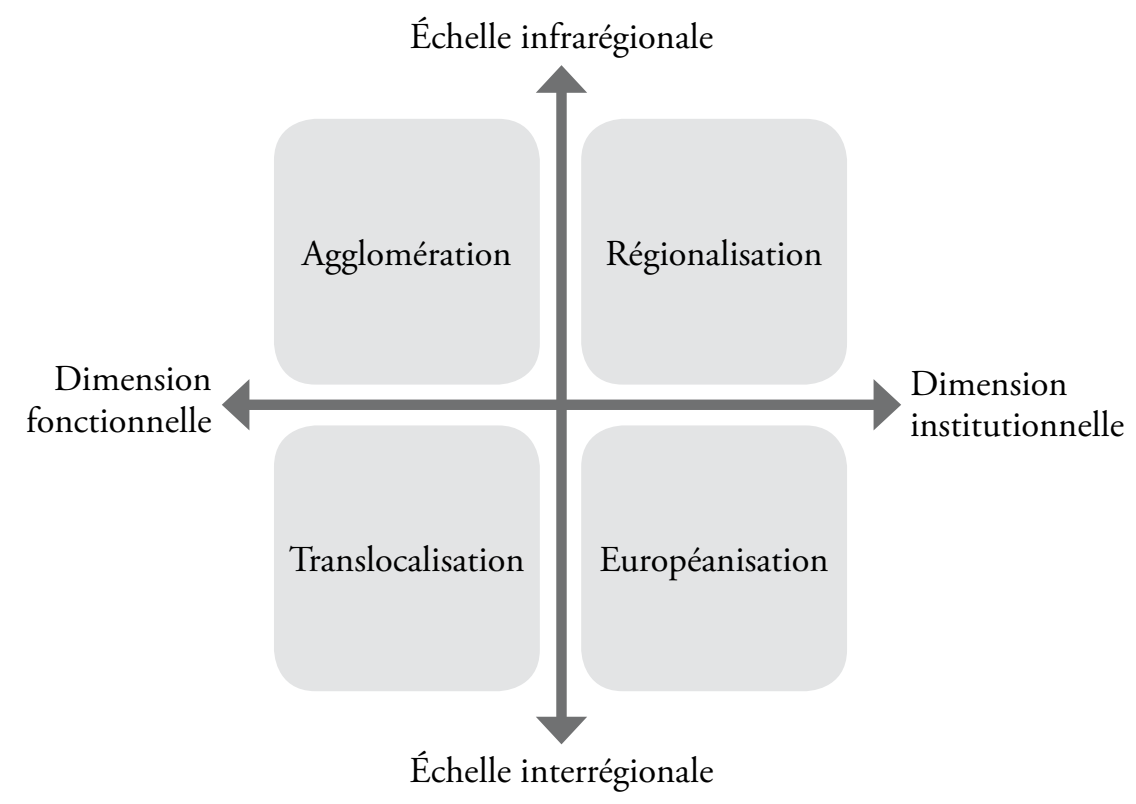

Figure 1 : La grille analytique ARTE montre la diversité des procédés au travers desquels les acteurs des régions périphériques nordiques prennent part aux processus d'innovation et de développement économique.

Source : Dubois et Carson 2016.

\section{Agglomération}

Le processus d'agglomération se réfère à l'évolution du paysage urbain (et donc rural) qui a eu lieu dans ces zones périphériques depuis la moitié des années 1990. L'essor démographique et économique d'une poignée de centres urbains, comme Umeå en Suède, Oulu en Finlande et Tromsø en Norvège, s'est déroulé en parallèle d'une certaine stagnation, voire déclin, économique ainsi que d'un vieillissement et décroissement démographique, dû au départ de jeunes femmes diplômées ou au retour de personnes retraitées, qui touche les zones moins accessibles et plus faiblement peuplées des zones intérieures. Ainsi, le paradoxe du développement territorial fait que ces régions, dans leur ensemble, deviennent à la fois plus urbanisées dans certaines parties déjà urbanisées et plus faiblement peuplées dans leurs zones les plus reculées ${ }^{13}$. Cette polarisation économique et démographique tend à concentrer les services et industries autour de ces pôles régionaux, ce qui change durablement l'équilibre interne de l'économie régionale. Un exemple intéressant

13 Ulf Wiberg, "Future Perspectives for Sparsely Populated Areas in Sweden ", in Changing Functions of Rural Areas in the Baltic Sea Region, J. Bañski (dir.), Varsovie, Polish Academy of Sciences, 2004, p. 95-107 ; Alexandre Dubois, Johanna Roto, « Making the Best of Europe's Sparsely Populated Areas. On Making Geographic Specificity a Driver for Territorial Development in Europe ", Nordregio Working Paper, 15, 2012. 
de cette polarisation économique concerne la distribution spatiale des industries manufacturières dans le Nord de la Suède qui étaient traditionnellement concentrées autour des sites miniers de l'intérieur, le long de la ligne Östersund-Kiruna, et qui se développent maintenant principalement autour des centres régionaux côtiers, notamment Umeå ${ }^{14}$.

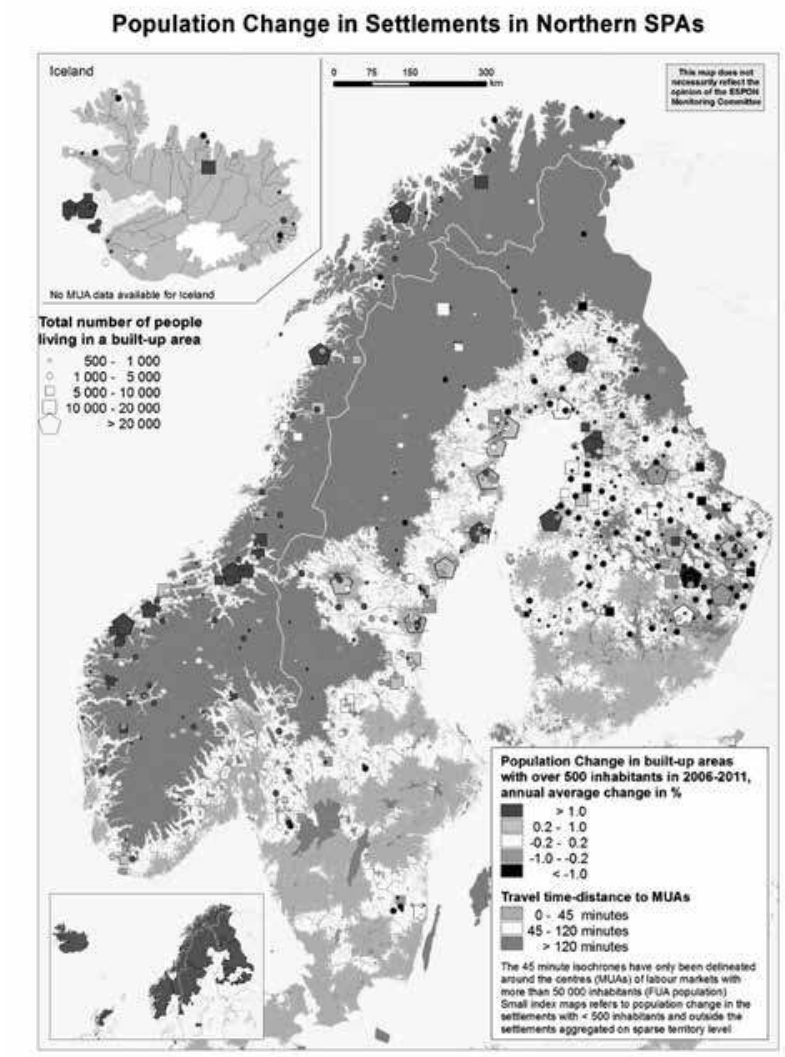

Figure 2 : La population des régions périphériques de Suède et de Finlande se concentre de plus en plus dans et autour de centres régionaux urbains, souvent en zone côtière (Umeå et Luleå en Suède ; Oulu et Vaasa en Finlande). À la polarisation démographique se joint une polarisation économique autour de ces mêmes centres régionaux qui sont pourvus d'universités et de centres de recherche pluridisciplinaires. Certains pôles intérieurs tels Östersund en Suède ou Rovaniemi et Joensuu en Finlande continuent néanmoins de croître. Les zones plus rurales de l'intérieur, d'où sont extraites les ressources naturelles, perdent de la population, et notamment les personnes jeunes et éduquées. Source : projet ORATE Geospecs www.espon.eu.

14 Alexandre Dubois, "Business Networks and the Competitiveness of Small Manufacturing Firms in Sweden's Northern Periphery ", Norsk Geografisk Tidsskrift. Norwegian Journal of Geography, 69, 3, 2015, p. 135-151. 


\section{Régionalisation}

Le processus de régionalisation prend en considération l'importance grandissante du niveau régional dans l'élaboration et l'implémentation des politiques publiques et, notamment, en lien avec la politique régionale européenne. Dans tous les pays nordiques, le niveau régional a eu historiquement un rôle assez faible puisqu'il est pris " en tenaille » entre un niveau local aux multiples prérogatives, notamment en ce qui concerne l'aménagement du territoire et les services de proximité, et un État providence fort ${ }^{15}$. En Suède, par exemple, le débat national sur la restructuration du niveau régional a duré de longues années et a abouti récemment à d'importantes réformes concernant la concentration de pouvoirs décisionnels attribués aux nouvelles entités administratives régionales, l'implémentation étant échelonnée depuis 2010 dans certaines régions pilotes du Sud et jusqu'à l'horizon 2019 pour le reste du pays. Les stratégies régionales de développement sont ainsi maintenant déléguées aux entités élues dans les 21 comtés. Pour les régions du Nord de la Suède, si cela apporte effectivement une plus grande capacité d'intégration des aspirations et préoccupations régionales et locales dans le schéma de programmation, cela crée aussi une situation inédite au niveau de la gouvernance régionale. Les centres régionaux deviennent ainsi des centres de décisions ayant un pouvoir grandissant sur les localités environnantes dites de "périphéries de ressources", notamment en ce qui concerne des investissements à long terme ayant trait à l'infrastructure physique et à celle de l'innovation. Cette régionalisation change donc l'équilibre des relations de pouvoir entre l'urbain et le rural au sein même de ces ensembles géographiques.

\section{Translocalisation}

Le processus de translocalisation correspond à une reconfiguration des relations entre acteurs économiques locaux et extralocaux qui a pu être observée ces dernières années. Étant gros producteurs de matières premières et ayant un marché domestique limité, les pays nordiques ont été traditionnellement très ouverts aux flux de la mondialisation, et notamment leurs " périphéries de ressources". Néanmoins, le rôle des périphéries nordiques comme pourvoyeur de ressources naturelles peu transformées les met en position défavorable ${ }^{16}$ au sein de chaînes de valorisation mondiales très hiérarchisées. Ainsi la translocalisation met en exergue une autre manière d'appréhender les échanges transnationaux fondée sur le développement de relations transversales d'interdépendance entre acteurs situés à des échelons plus bas des filières économiques, et notamment les $\mathrm{PME}^{17}$. Ces

15 Lisa Hörnström, "Strong Regions within the Unitary State : The Nordic Experience of Regionalization ", Regional \& Federal Studies, 23, 4, 2013, p. 427-443.

16 Nathan Young, "Globalization from the Edge : A Framework for Understanding How Small and Medium-Sized Firms in the Periphery "Go Global" ", Environment and Planning. A, 42, 4, 2010, p. $838-855$.

17 Alexandre Dubois, "Transnationalising Entrepreneurship in a Peripheral Region : The Translocal Embeddedness Paradigm ", Journal of Rural Studies, 46, 2016, p. 1-11. 
relations ne s'appuient plus seulement sur une approche en termes de transactions, mais aussi sur le développement de nouveaux liens de collaboration entre acteurs de différentes localités fondés sur la réciprocité et la mutualité et non plus la dépendance ou la domination. Dans le Nord de la Suède, plusieurs études ont aussi montré l'impact local grandissant des migrations à petite échelle européenne $^{18}$ ou asiatique ${ }^{19}$ dans l'adoption de nouveaux modes de fonctionnement de l'entreprenariat local. En effet, ces migrations permettent de revitaliser certains villages en développant des activités entrepreneuriales, en dehors des industries d'exploitation des ressources naturelles. Ces entrepreneurs-migrants utilisent leurs réseaux sociaux transnationaux afin de développer leur activité économique, souvent dans les zones rurales sujettes à la dépopulation ${ }^{20}$, qui ainsi accompagne une transition des économies locales fondée sur une plus grande capacité des acteurs locaux à participer à des échanges avec des acteurs situés en dehors des limites de l'économie locale.

\section{Européanisation}

Enfin, l'européanisation a été fortement associée à la notion de territorialité, liée au positionnement des territoires européens, et notamment les plus périphériques, dans un ensemble socioéconomique et institutionnel plus vaste que celui de l'État-nation autour de nouvelles valeurs partagées ${ }^{21}$. L'accession de la Finlande et de la Suède à l'Union européenne en 1995 a ainsi permis aux acteurs régionaux d'initier un " désenclavement institutionnel » en nouant un dialogue à l'échelon européen concernant leurs aspirations de développement, notamment en les raccordant aux discours et objectifs de développement de l'Union dans son ensemble, ayant trait à l'innovation, la compétitivité économique ou la cohésion territoriale, telles que promues par la stratégie Europe 2020 ou l'Agenda territorial 2020. Une manifestation de ce nouveau paysage institutionnel transnational auquel les régions septentrionales participent est l'établissement du réseau $\mathrm{NSPA}^{22}$ promouvant les intérêts collectifs d'une dizaine de régions périphériques finlandaises, norvégiennes et suédoises. Le réseau NSPA est une collaboration volontaire créée par les régions elles-mêmes afin d'avoir plus de poids dans leur dialogue avec les acteurs européens tels que la Commission européenne, le Parlement européen ou le Comité des régions. Enfin, la participation aux programmes

18 Marco Eimermann, «Ambivalent Dutch Lifestyle Migrants in Rural Sweden », AEMI Journal, 12, 2014, p. 48-57.

19 Natasha A. Webster, Karen Haandrikman, "Thai Women Entrepreneurs in Sweden : Critical Perspectives on Migrant Small Businesses », Women's Studies. International Forum, 60, 2017, p. 17-27.

20 Charlotta Hedberg, Gunnell Forsberg, Ali Najib, "When the World Goes Rural : Transnational Potentials of International Migration in Rural Swedish Labour Markets », in Translocal Ruralism. Mobility and Connectivity in European Rural Spaces, C. Hedberg et R. M. do Carmo (éd.), Dordrecht, Springer, 2012, p. 125-142.

21 Matti Fritsch, "Interfaces of European Union Internal and External Territorial Governance : The Baltic Sea Region ", in Territorial Development, Cohesion and Spatial Planning. Building on EU Enlargement, N. Adams, G. Cotella et R. Nunes (dir.), Londres, Routledge, 2010, p. 382-401.

22 Acronyme anglais de Northern Sparsely Populated Areas. 
de coopération transfrontaliers et interrégionaux Interreg, fondés sur des échanges au niveau opérationnel et stratégique entre acteurs régionaux et financés par les fonds structurels européens, a permis aux régions périphériques de se " désenclaver » en nouant de nouvelles alliances et en fondant de nouvelles relations avec d'autres régions européennes en créant un projet de développement commun, notamment au sein de la région baltique.

\section{La matrice ARTE appliQuée À L'Étude d’une PME de Malå (SuÈde) ${ }^{23}$}

Dans cette section, nous allons utiliser les quatre éléments de la matrice ARTE afin de mieux comprendre la trajectoire de développement d'une PME spécialisée dans la fabrication de radars pour l'exploration souterraine et basée à Malå, une localité minière (cuivre essentiellement) de la région suédoise de Västerbotten dont la plupart des mines sont aujourd'hui fermées. Nous allons plus particulièrement nous intéresser à la période entre 1994 et 2008, durant laquelle l'actionnariat de l'entreprise était détenu par une dizaine d'employés après l'avoir rachetée à l'État suédois pendant la vague de privatisation des années 1990.

En effet, lors de cette période d'actionnariat local, nombre d'initiatives ont été prises afin de mobiliser un vaste réseau de collaborations tant au niveau régional qu'à l'international. Au niveau régional, la PME a développé des relations privilégiées avec une demi-douzaine de fournisseurs, ce qui lui permet de disposer d'un système de production local pour garder une certaine réactivité aux aléas du marché (commandes urgentes, test de nouveaux produits) et une flexibilité dans sa chaîne de production et de maintenir un savoir-faire industriel stratégique dans la région. La PME s'est aussi engagée avec d'autres acteurs régionaux de la filière minière dans le développement d'une plate-forme de coopération, GEORANGE, permettant de coordonner, dans une certaine mesure, leurs stratégies de développement et de peser comme acteur économique collectif. Au niveau international, la PME a cherché à trouver de nouveaux débouchés pour l'utilisation de ses radars, c'est-à-dire en dehors du traditionnel secteur minier. Durant cette période, l'introduction de nouveaux modèles de radar aux performances et à la maniabilité accrues a permis à la PME de se positionner dans des domaines de la recherche nécessitant l'exploration souterraine, comme l'archéologie, mais pour des applications ayant trait à la découverte de couches aquifères. La PME a ensuite cherché à valoriser ce positionnement à l'international dans un secteur de haut niveau en établissant en 1997 une filiale dans la région du triangle universitaire de Caroline du Nord aux États-Unis. Cette présence physique permettra d'instaurer des relations de proximité dans le secteur de la recherche. Enfin, la PME a aussi développé des réseaux au niveau européen en participant à de

23 Pour une étude plus approfondie de ce cas, voir l'article (en anglais) d'Alexandre Dubois, Dean B. Carson, "The Relational Geography of Post-Staples Development : A Case in Malå, North Sweden ", Journal of Rural and Community Development, à paraître. 
nombreux projets de recherche financés par l'Union européenne ainsi qu'à un réseau connectant des acteurs dans les principales régions minières européennes.

La trajectoire de cette PME lors de la période d'actionnariat local illustre comment une mobilisation stratégique de multiples réseaux peut permettre à des acteurs précédemment tournés vers des industries de ressources naturelles de se positionner dans de nouveaux secteurs et de nouveaux marchés. Bien que cette transition économique " post-ressources naturelles " implique souvent une internationalisation accrue, le cas de notre PME montre aussi qu'une telle transition doit s'accompagner, pour être durable dans le temps, de nouvelles formes de coopération entre acteurs régionaux.

\section{LA TRANSITION ÉCONOMIQUE DES RÉGIONS PÉRIPHÉRIQUES : L'EXEMPLE DE LA « SPÉCIALISATION INTELLIGENTE »}

Une illustration contemporaine de cette recherche d'une transition économique est l'implémentation actuelle des stratégies régionales d'innovation dites de la "spécialisation intelligente ${ }^{24}$. La spécialisation intelligente promeut l'émergence de nouveaux domaines économiques issus préférablement du tissu économique existant. Elle diffère des vagues précédentes de stratégies régionales d'innovation par le fait qu'elle ne tend à soutenir ni un secteur économique ni un petit nombre d'entreprises, " championnes régionales " de l'innovation en particulier. Un obstacle souvent mentionné à l'implémentation de la spécialisation intelligente dans les régions périphériques est leur manque de masse critique d'agents économiques ${ }^{25}$. Les acteurs régionaux eux-mêmes perçoivent ce manque de masse critique et la surdépendance aux ressources naturelles comme des obstacles structurels à son implémentation ${ }^{26}$.

Le défi majeur lié à l'implémentation de la spécialisation intelligente dans les régions périphériques nordiques vient du fait qu'elle doit intégrer la problématique de la gouvernance des ressources naturelles à celle, plus classique, des systèmes régionaux d'innovation, les espaces économiques et les acteurs impliqués dans ces deux processus étant souvent distincts : d'un côté, des processus d'innovation et des industries à haute valeur ajoutée (high tech, services) se développant dans les pôles régionaux; de l'autre, des industries d'exploitation des ressources naturelles toujours très profitables économiquement et localisées de façon dispersée dans les zones plus faiblement peuplées de l'intérieur. Mais cette double problématique pose aussi la question de la temporalité des stratégies régionales de développement. En effet, l'exploitation des ressources naturelles est toujours très profitable à ce jour et permet de générer d'importants revenus pour les communautés locales ainsi que pour l'État. Par conséquent, il apparaît peu concevable, à court

24 Jukka Teräs, Alexandre Dubois, Jens Sörvik, Martina Pertoldi, «Implementing Smart Specialisation in Sparsely Populated Areas ", S3 Working Paper, 10, 2015.

25 Dominique Foray, Smart Specialisation. Opportunities and Challenges for Regional Innovation Policy, Londres, Routledge, 2014.

26 Jukka Teräs, Alexandre Dubois, Jens Sörvik, Martina Pertoldi, op. cit., p. 8. 
et même à moyen terme, que ces régions se passent de ces industries. Pour les autorités régionales, il faut donc permettre à ces industries de fonctionner à plein tout en préparant le futur du développement régional qui se conjugue autour de l'économie de la connaissance, comme dans n'importe quelle autre région nordique. L'économie de la connaissance est déjà bien établie dans ces régions, mais ses acteurs sont surtout concentrés dans les centres régionaux les plus importants tels Umeå en Suède, Oulu en Finlande ou Tromsø en Norvège. En effet, ces villes sont dotées d'université et d'instituts de recherche multidisciplinaires ainsi que d'une multitude d'incubateurs d'entreprises innovantes qui profitent essentiellement au dynamisme des secteurs de services et technologiques en toute proximité. Surtout, afin de maintenir leur compétitivité internationale ces centres sont plus enclins à s'engager dans des réseaux d'excellence scientifiques et technologiques, notamment au niveau européen. Ainsi, on peut considérer que bien que l'économie de la connaissance soit fortement ancrée dans ces régions, elle est, géographiquement, très concentrée dans les pôles urbains régionaux et participe donc peu à la diversification des économies des " périphéries des ressources ». La problématique du développement territorial dans les régions périphériques est donc tout autant géographique et sectorielle que liée à la capacité à mobiliser des connaissances permettant de promouvoir la compétitivité des industries traditionnelles et d'entreprises plus innovantes.

L'étude des processus d'élaboration et d'implémentation des stratégies régionales de la "spécialisation intelligente " permettent de mieux comprendre comment les régions périphériques nordiques conjuguent au futur cette problématique du développement territorial. Par exemple, le développement d'un domaine de l'industrie verte dans la région norvégienne du Nordland permet non seulement de créer de nouvelles opportunités économiques liées à de nouveaux modes de production et de consommation d'énergies renouvelables, mais aussi une vision collective du futur du territoire régional autour de nouvelles valeurs " globales ", notamment liées à l'environnement. Dans la région du Nordland, le développement de l'éolien illustre la nécessité de penser ces projets comme un processus de négociation multiacteur. En effet, les investisseurs de ces projets sont souvent les entreprises régionales d'énergie hydroélectrique alors que le déploiement des infrastructures affecte directement les intérêts du peuple indigène Sami et du secteur de l'élevage de rennes ${ }^{27}$.

La création de nouveaux domaines économiques est ainsi liée à une exploitation plus efficace et durable des ressources naturelles régionales s'appuyant sur le développement de nouvelles techniques et technologies propres, mais aussi sur la capacité des acteurs à créer de nouvelles applications industrielles et de nouveaux débouchés économiques, notamment à l'international. Ces stratégies permettent d'allier l'héritage industriel et culturel de ces régions à des procédés d'innovation régionale plus classiques.

27 Åge Mariussen, Arild Gjertsen, Bjarne Lindeløv, Jarle Løvland, Challenges for Smart Strategies in Resource Based Regions. The Case of Wind Energy in Nordland, Bodø, Nordlandsforskning, 2014. 
L'implémentation des stratégies de la spécialisation intelligente repose aussi sur la participation de la société civile, notamment des associations diverses (riverains, consommateurs ou environnementales) ou des entreprises et organisations provenant de l'économie sociale et solidaire. Ce type de structures est donc fondé sur des échanges plus ouverts et informels que dans le cadre plus formalisé des partenariats public-privé ou de la coopération recherche-public-privé connu sous le nom de Triple Helix. Ainsi dans la région septentrionale suédoise de Västerbotten, plusieurs initiatives ont été établies afin de permettre aux différents acteurs du développement territorial de se rencontrer et d'établir de nouveaux liens. Le Forum régional de l'innovation est pensé comme une future plate-forme permettant les échanges d'idées et l'incubation de projets innovants à l'échelle régionale. L'initiative " Meeting point Lycksele " est une plate-forme du même genre, mais circonscrite aux acteurs de la municipalité de Lycksele. Outre ces points de rencontre physiques, bien qu'occasionnels, la région Västerbotten s'appuie aussi sur un réseau d'organisations publiques, telles que ALMI Nord ou Uminova, dont le rôle est de faciliter la mise en réseau des entreprises, essentiellement des PME, et de promouvoir les échanges de connaissances permettant le développement de coalitions d'acteurs ouvertes et temporaires de type " projet ".

En revanche, comme le suggère l'exemple de la Laponie finlandaise, ce type de stratégie régionale d'après une vision interactive de l'innovation est dépendant de la volonté des acteurs régionaux de s'y impliquer. Ainsi, dans cette région à forte tradition minière et forestière, les autorités régionales reconnaissent une certaine réticence de la part des acteurs industriels régionaux à s'impliquer dans ce processus. En outre, dans le cas de la Laponie, un défi d'envergure de cette stratégie est de pouvoir accommoder les intérêts de différents secteurs clés, comme l'industrie minière et le tourisme, qui ont leur propre logique dans l'exploitation du capital naturel de la région et souvent des intérêts divergents, afin que le développement de l'un ne se fasse pas aux dépens de l'autre. La stratégie régionale de la Laponie soulève aussi la question de son inclusion dans la stratégie de développement de l'espace arctique, mettant ainsi en avant l'importance pour ces régions d'insérer leurs problématiques et prospectives de développement propres dans celles d'un ensemble territorial plus large.

\section{Conclusions}

Cet article souhaitait donner une vision d'ensemble des problématiques liées au développement territorial dans les régions périphériques nordiques. En schématisant la trajectoire historique de ce développement, on peut ainsi dire que deux systèmes territoriaux et économiques se sont développés au fil des années : l'un fondé sur l'exploitation des ressources naturelles, localisé dans les vastes étendues peu habitées de l'intérieur de ces pays, et l'autre fondé sur une économie de services et de la connaissance, concentrée géographiquement au sein de quelques centres urbains régionaux. Bien que tous les deux très ouverts aux relations 
extérieures, les liens fonctionnels entre ces deux sous-systèmes ont été historiquement assez faibles en dépit leur appartenance au même ensemble géographique ${ }^{28}$. Ainsi, une transition économique liant une meilleure gouvernance des ressources naturelles à une économie de la connaissance passe nécessairement par une reconfiguration des liens fonctionnels et institutionnels entre zones urbanisées et zones rurales dites des "périphéries des ressources".

L'articulation de ces deux systèmes passe premièrement par le développement d'une vision commune de l'avenir de l'économie régionale. De ce point de vue-là, les premières ébauches de stratégies de la "spécialisation intelligente ", bien que perfectibles et assez peu prospectives en fin de compte, offrent une telle plateforme dans laquelle chaque acteur doit se projeter au-delà du développement de son propre secteur d'activité. Mais cette articulation doit aussi passer par un stade opérationnel qui cherche à établir des projets communs concrets nécessitant de mutualiser leurs ressources, même de façon temporaire. Ici, la création de plates-formes de proximité géographique temporaire ${ }^{29}$ (forums, salons, ateliers...) permet de promouvoir des échanges entre acteurs ne nécessitant pas leur relocalisation permanente. Ces types d'initiatives ponctuelles dans le temps et dans l'espace sont utilisés systématiquement dans les régions périphériques afin de pallier les longues distances internes qui empêchent les acteurs de divers secteurs de se rencontrer de façon impromptue. Dans ce cadre-là, l'enjeu d'une démarche de prospective territoriale est donc de promouvoir la complémentarité entre ces systèmes, au contraire d'une dépendance de l'un envers l'autre. Le défi pour l'implémentation de stratégies régionales de développement consiste donc à trouver un nouvel équilibre territorial entre les besoins et potentiels de ces zones aux dynamiques divergentes.

En outre, le positionnement de ces espaces régionaux dans des ensembles géographiques plus larges permet de minimiser l'effet d'enclavement que leur position géographique périphérique, tant au niveau national qu'européen, peut générer. Ainsi ces espaces régionaux qui pourraient être considérés comme étant "à la marge $~_{30}$ ne sont pas pour autant des espaces marginalisés. C'était le propos principal de cet article. Par le biais d'une attitude volontariste, symbolisé par l'intense travail de lobbying de l'association NSPA notamment, ces régions ont su se positionner non plus comme espace-frontière ${ }^{31}$, mais plutôt comme espaceinterface au croisement de plusieurs ensembles territoriaux supranationaux tels que l'Union européenne, mais aussi la région nordique, la région baltique et l'espace arctique.

28 Dean B. Carson, Doris A. Carson, "Local Economies of Mobility in Sparsely Populated Areas : Cases from Australia's Spine ", Journal of Rural Studies, 36, 0, 2014, p. 340-349.

29 André Torre, "Retour sur la notion de proximité géographique ", Géographie, Économie, Société, 11, 1, 2009, p. 63-75.

30 Erik Gløersen, op. cit., p. 2.

31 Ibid. 